\title{
Prefrontal and parietal cortex in human episodic memory: an interference study by repetitive transcranial magnetic stimulation
}

\author{
Simone Rossi, ${ }^{1,2}$ Patrizio Pasqualetti, ${ }^{3}$ Giancarlo Zito, ${ }^{3}$ Fabrizio Vecchio, ${ }^{3,4}$ Stefano F. Cappa, ${ }^{5}$ Carlo Miniussi, ${ }^{2,6}$ \\ Claudio Babiloni ${ }^{2,4}$ and Paolo M. Rossini $i^{2,3,7}$ \\ ${ }^{1}$ Dipartimento di Neuroscienze, Sezione Neurologia, Università di Siena, Policlinico le Scotte, Viale Bracci, I-53100, Siena, Italy \\ 2Unità di Neuroscienze Cognitive, IRCCS S. Giovanni di Dio, AFaR, Via Pilastroni 4, I-25125, Brescia, Italy \\ ${ }^{3}$ AFaR.- Dipartimento di Neuroscienze, S. Giovanni Calibita, Fatebenefratelli Isola Tiberina, I-00186, Roma, Italy \\ ${ }^{4}$ Dipartimento di Fisiologia Umana e Farmacologia, Università La Sapienza, P.le Aldo Moro 5, I-00185, Roma, Italy, and CONI \\ Servizi, Roma, Italy \\ ${ }^{5}$ Department of Neuroscience, Vita-Salute University and S. Raffaele Scientific Institute, DIBIT, Via Olgettina 58, I-20132, Milano, Italy \\ ${ }^{6}$ Dipartimento di Scienze Biomediche e Biotecnologie, Università di Brescia, Italy \\ ${ }^{7}$ Clinica Neurologica, Università Campus Biomedico, Via Longoni 71, Roma, Italy
}

Keywords: long-term memory, neuroimaging, parietal cortex, prefrontal cortex, rTMS, transcranial magnetic stimulation

\section{Summary}

Neuroimaging findings, including repetitive transcranial magnetic stimulation (rTMS) interference, point to an engagement of prefrontal cortex (PFC) in learning and memory. Whether parietal cortex (PC) activity is causally linked to successful episodic encoding and retrieval is still uncertain. We compared the effects of event-related active or sham rTMS (a rapid-rate train coincident to the very first phases of memoranda presentation) to the left or right intraparietal sulcus, during a standardized episodic memory task of visual scenes, with those obtained in a fully matched sample of subjects who received rTMS on left or right dorsolateral PFC during the same task. In these subjects, specific hemispheric effects of rTMS included interference with encoding after left stimulation and disruption of retrieval after right stimulation. The interference of PC-rTMS on encoding/retrieval performance was negligible, lacking specificity even when higher intensities of stimulation were applied. However, right PC-rTMS of the same intensity lengthened reaction times in the context of a purely attentive visuospatial task. These results suggest that the activity of intraparietal sulci shown in several funtional magnetic resonance studies on memory, unlike that of the dorsolateral PFC, is not causally engaged to a useful degree in memory encoding and retrieval of visual scenes. The parietal activations accompanying the memorization processes could reflect the engagement of a widespread brain attentional network, in which interference on a single 'node' is insufficient for an overt disruption of memory performance.

\section{Introduction}

Episodic memory is a complex set of human cognitive processes that allows the encoding, storage and intentional recollection (retrieval) of unique events associated with the context in which they occurred (Baddeley et al., 2001). Therefore, correct functioning of encoding/retrieval mechanisms is vital in forming the conscious story of our existence (Tulving, 2002). Studies in neurological patients indicate that episodic memory is dramatically disrupted by lesions of the medial temporal lobe, and in particular of the hippocampal formation and enthorinal cortex (Squire et al., 2001), whereas lesions in other brain areas - such as the prefrontal cortex (PFC) - are associated only with more subtle dysfunctions of learning and memory (Alexander et al., 2003). Lesions in other neocortical areas, such as the parietal lobe, appear to induce material-specific effects, largely limited to the short-term retention of information. Left-sided lesions are associated with defective performance in auditory-verbal short-term memory

Correspondence: Dr S. Rossi, ${ }^{1}$ Dipartimento di Neuroscienze, as above.

E-mail: Rossisimo@unisi.it

Received 8 July 2005, revised 28 October 2005, accepted 23 November 2005 tasks, and impaired spatial span performance has been found in patients with right parietal damage (De Renzi \& Nichelli, 1975). Rare cases of 'retrosplenial amnesia' have been described following lesions of parietal midline structures (Valenstein et al., 1987).

Other relevant evidence for the role of neocortical regions in episodic memory comes from converging neuroimaging studies in normal subjects: techniques measuring cerebral blood flow or metabolism (positron emission tomography and functional magnetic resonance), magnetoelectric brain activity [electroencephalography (EEG)/magnetoencephalography] and event-related cortical activity (event-related potentials) are increasingly unveiling the functional correlates of human learning and memory. Overall, these studies have indicated that regional activity co-varies with encoding and retrieval tasks in several brain regions, therefore suggesting the existence of a distributed neural network governing this highly specialized process. The main functional 'nodes' of such a network include the PFCs, the medial temporal lobes, but also the parietal cortices (PCs) and precuneus (for extensive reviews see Buckner \& Wheeler, 2001; Fletcher \& Henson, 2001; Rugg et al., 2002; Cabeza et al., 2003; Simons \& Spiers, 2003; Wagner et al., 2005), often functionally 
coupled in complex combinations (Cabeza \& Nyberg, 2000; Rugg et al., 2002; Kesner \& Rogers, 2004; Wagner et al., 2005). The involvement of parietal areas is suggested by several neuroimaging and electrophysiological studies of visuospatial and verbal episodic memory (for reviews see Cabeza \& Nyberg, 2000; Rugg et al., 2002; Wagner et al., 2005), although in only a few of these was PC activity specifically addressed (Henson et al., 1999; Mottaghy et al., 1999; Konishi et al., 2000; Cabeza et al., 2003; Hayes et al., 2004; Mayes et al., 2004).

However, neuroimaging studies, being correlational in nature, cannot disentangle whether task-related activations are truly necessary for task completion or if they are simply associated with performance. This can be better approached by applying repetitive transcranial magnetic stimulation (rTMS), a unique technique which allows us to produce, in vivo, a brief and reversible decline of the performance only if the stimulated area is causally engaged in the task under investigation (Walsh \& Cowey, 2000; Walsh \& Pascual-Leone, 2003; Rossi \& Rossini, 2004). The interferential approach with event-related rTMS is increasingly used across different labs to investigate hemispheric specializations of PFCs during long-term memorization tasks concerning both visuospatial and verbal cues (Rossi et al., 2001, 2004; Miniussi et al., 2003; Rami et al., 2003; Sandrini et al., 2003; Floel et al., 2004; Kohler et al., 2004).

A main common finding in young adults who received rTMS on the dorsolateral PFCs (DLPFCs) was the direct confirmation of the functional specialization of these areas in episodic visuospatial memory (see Fletcher \& Henson, 2001). The left DLPFC has been shown to be prevalent during encoding and the right DLPFC during retrieval of visuospatial memoranda (Rossi et al., 2001, 2004), according to the neuroimaging-based HERA (Hemispheric Encoding Retrieval Asymmetry) model (Habib et al., 2003). By using the same set of visuospatial stimuli as previous studies (Rossi et al., 2001, 2004), a recent high-resolution EEG investigation of cortical rhythmicity during episodic memory highlighted a functional frontoparietal connectivity by showing that the HERA predictions were fitted only by high-frequency oscillations in the gamma band (30$45 \mathrm{~Hz}$ ), the amplitude of which was modulated in PC rather than PFC (Babiloni et al., 2004). However, an ad-hoc analysis based on the direct transfer function method, which reflects the 'direction' of the information flow between distant but functionally connected cortical sites, successively revealed that a fronto-parietal coherence at the gamma band prevailed in the left hemisphere during the encoding and in the right hemisphere during the retrieval (Babiloni et al., 2006), in line with the prefrontal HERA model prediction (Habib et al., 2003).

The aim of the current event-related rTMS study is to address directly the functional relevance of the activations described in PCs during episodic encoding and retrieval. The intraparietal sulcus was targeted to this end because this region was previously found to be engaged consistently in episodic encoding and retrieval (for reviews see Mottaghy et al., 1999; Cabeza et al., 2003; Fujii et al., 2004;
Hayes et al., 2004; Mayes et al., 2004; Wagner et al., 2005). Moreover, our aim was to contrast results of PC interference with those obtained in a fully comparable sample of subjects who received rTMS on the DLPFCs during the same task and showed clear-cut behavioural consequences (Rossi et al., 2001, 2004). Of note, the functional relevance of the PC along episodic encoding and retrieval has not yet been directly addressed by rTMS interference. Additional control experiments included active and sham rTMS of the PC with higher intensity and active and sham rTMS of the same regions during a purely visuospatial attentive task.

\section{Subjects and methods \\ Subjects}

Forty-two healthy volunteers took part in the study. They gave their written informed consent after approval of the protocol by the local Ethics Committee. Their medical history and objective neurological examination were normal, and they had never taken neuroactive drugs. No other significant medical conditions were present.

The core of the study was to compare the behavioural effects of rTMS delivered to the DLPFC and the PC on a standardized episodic memory task of visual scenes. To this end, two groups of ten subjects were examined (Table 1): those of the first group received active or sham rTMS to the left and right PC ('PC-rTMS group', see later for details). These were matched $1: 1$ with subjects of a second group, retrospectively selected among a cohort of 66 individuals already tested with the same memory task during active or sham rTMS on left and right DLPFC ('PFC-rTMS group', Rossi et al., 2001, 2004). The matching procedure took into account mainly the baseline level of performance in the memory task under investigation (hits-false alarms of the Baseline block). When a subject of the PC-rTMS group could have been matched to more than one subject of the PFC-rTMS group, handedness, gender and age were considered as additional matching factors. These procedures resulted in the statistics reported in Tables 1 and 2.

Subjects were studied while comfortably sitting on a reclining chair, with their head stabilized, in front of a 17-inch monitor. They kept their forearms resting on armchairs, with their right index finger resting between two buttons spaced $6 \mathrm{~cm}$ apart. Ten minutes of training, performed with a different set of pictures, allowed the subjects to practice the task, and with both sham or active rTMS prior to the actual experimental session.

\section{Experimental conditions}

The memory task was the same previously utilized in EEG (Babiloni et al., 2004, 2006) and rTMS (Rossi et al., 2001, 2004) event-related experimental settings. Briefly (see Fig. 1a), the 'indoor-outdoor paradigm' included six blocks of encoding followed by six blocks of

TABLE 1. Demographics of subjects who received rTMS on prefrontal and parietal cortex

\begin{tabular}{|c|c|c|c|c|c|}
\hline & $\begin{array}{l}\text { PFC } \\
(n=10)\end{array}$ & $\begin{array}{l}\text { PC- } 90 \% \\
(n=10)\end{array}$ & $\begin{array}{l}\text { PC- } 120 \% \\
(n=10)\end{array}$ & Test applied & $P$-value \\
\hline Age (years) & $28.7 ; 2.3$ & $27.3 ; 1.9$ & $28.6 ; 1.5$ & ANOVA & $>0.20$ \\
\hline Gender $(\mathrm{F}$ and $\mathrm{M})$ & $3 \mathrm{~F}$ and $7 \mathrm{M}$ & $4 \mathrm{~F}$ and $6 \mathrm{M}$ & $4 \mathrm{~F}$ and $6 \mathrm{M}$ & Chi-squared & $>0.20$ \\
\hline Handedness (median)* & $93 \%$ & $90 \%$ & $90 \%$ & Kruskal-Wallis & $>0.20$ \\
\hline Handedness (range)* & $50-100 \%$ & $45-100 \%$ & $50-100 \%$ & & \\
\hline
\end{tabular}

Group PFC, subjects who received rTMS on the dorsolateral prefrontal cortex; groups PC, subjects who received rTMS on the parietal cortex at $90 \%$ or at $120 \%$ of individual motor thresholds. *Values are median and range percentages of the Oldfield's score. Ages are given as means $\pm \mathrm{SD}$. 
a) Experimental design 1 hour

\begin{tabular}{|c|c|c|}
\hline \multirow[b]{2}{*}{$\begin{array}{l}\text { Blocks } \\
\text { R-Enc } \\
\text { L-Enc }\end{array}$} & \\
\hline & $\begin{array}{l}\text { rTMS in } \\
\text { Encoding } \\
\text { right IPS } \\
\text { left IPS } \\
\end{array}$ & $\begin{array}{l}\text { rTMS in } \\
\text { Retrieval } \\
\text { no } \\
\text { no } \\
\end{array}$ \\
\hline Sham & left IPS & right IPS \\
\hline $\begin{array}{l}\text { R-Ret } \\
\text { L-Ret }\end{array}$ & $\begin{array}{l}\text { no } \\
\text { no }\end{array}$ & $\begin{array}{l}\text { right IPS } \\
\text { left IPS }\end{array}$ \\
\hline Baseline & no & no \\
\hline
\end{tabular}

b) Experimental timing

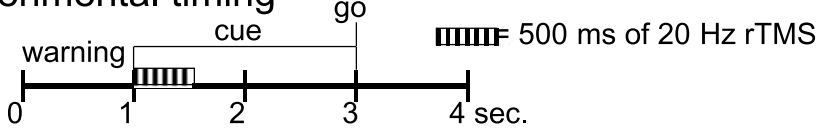

c) Site of stimulation

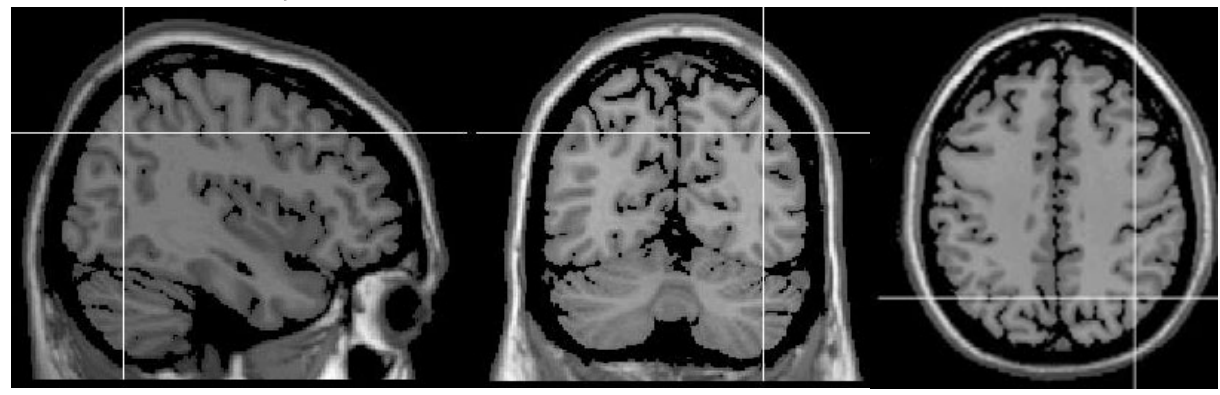

FIG. 1. Experimental details. (a) The experimental conditions. The six encoding/retrieval blocks were: R-Enc, right rTMS in encoding, no stimulation in retrieval; L-Enc, left rTMS in encoding, no stimulation in retrieval; Sham, sham rTMS (left in encoding and right in retrieval); R-Ret, right rTMS in retrieval, no stimulation in encoding; L-Ret, left rTMS in retrieval, no stimulation in encoding; Baseline, absence of stimulation. The shaded rectangles contain the 'reference' blocks (i.e. Sham and Baseline). The same block design was used for subjects who received rTMS at parietal and prefrontal sites. (b) Timing of warning, cue and go stimuli and of the rTMS train with respect to the cue. (c) Site of stimulation. MRI of a subject showing the sagittal (left), coronal (middle) and axial (right) projections on the cortical surfaces of stimulation sites in the right (P4) hemisphere, corresponding to the intraparietal suclus [Talaraich coordinates of P4 were: 40, -62 , 40; Talaraich coordinates of the homologous right stimulation site P3 (not shown) were: $-43,-64,39]$. The intercept of the coordinates (white lines) corresponded to BA 39 bilaterally.

retrieval, pseudo-randomized and counterbalanced between subjects as order of presentation. For each block of the encoding phase, 16 complex coloured magazine pictures (eight indoor and eight outdoor scenes) were randomly presented on the monitor for $2 \mathrm{~s}$, with an intertrial interval of $10 \mathrm{~s}$. Images were preceded by a visual warning stimulus (a red spot lasting $1 \mathrm{~s}$ ). Subjects were instructed to press, with their right index finger, one of the two buttons (left, indoor; right, outdoor) after the presentation of a green circle in the middle of each picture (the 'go' signal), which appeared $1 \mathrm{~s}$ after the picture presentation.

The six encoding blocks had six corresponding retrieval blocks $(1 \mathrm{~h}$ later), each containing 16 pictures of indoor scenes randomly presented: eight of them had been seen previously (tests) and eight were novel (distractors). The timing of warning and go signals, picture presentation and intervals were as for the encoding. Subjects were again asked, in a yes-no recognition task, to answer by pressing one of the two buttons (left, 'old' or test; right, distractor) after the go stimulus. Thus, the protocol excluded an overt working memory load, as pictures were presented on the screen until the subject's response. Subjects were familiarized with the task (with a different set of pictures) and with left and right rTMS (both active and sham) for about $10 \mathrm{~min}$ before starting the actual experiment. The six encoding/retrieval blocks were labelled according to the type (active or sham) and the side (left/right) of the rTMS applied on the PCs (see Fig. 1 for the complete labelling of the experimental conditions).

\section{Stimulation and identification of the target regions}

rTMS was delivered through a focal, figure-of-eight coil (outer diameter of each wing $7 \mathrm{~cm}$ ) connected with a standard Mag-Stim Super Rapid stimulator (maximum output $2.2 \mathrm{~T}$ ). As previously detailed (Rossi et al., 2001, 2004), individual resting excitability thresholds for left and right motor cortex stimulation were first determined by using the same coil and stimulator; afterwards, the intensity was reduced by $10 \%$.

Left and right PCs were stimulated by placing the anterior end of the junction of the two coil wings on P3 or P4 (10-20 international EEG system), respectively. A mechanical arm maintained the handle of the coil angled backward at about $45^{\circ}$ away from the midline. This position was marked on a transparent bathing cuff firmly adherent to the scalp. This allowed the correct repositioning of the coil prior to the appearance of each warning signal.

P3 and P4 locations on the subject's scalp were automatically identified using the SofTaxic Navigator system, on the basis of digitized skull landmarks (nasion, inion and two pre-auricular points) and about 40 scalp points (Fastrak Polhemus digitizer). Individual magentic resonance images were available for three subjects (see Fig. 1c); in the remaining subjects, Talairach coordinates of cortical sites underlying P3 and P4 locations were automatically estimated by the SofTaxic Navigator Stereotaxic Navigator System (E.M.S. Italy, http://www.emsmedical.net), on the basis of an MRI-constructed 
stereotaxic template (estimated accuracy of $\pm 0.5 \mathrm{~cm}$, Talairach space). These target regions (corresponding to the scalp projection of left and right intraparietal suclus) were chosen on the basis of independent neuroimaging studies that consistently showed an engagement of such brain areas in episodic encoding and retrieval (see Wagner et al., 2005).

Trains of rTMS $(90 \%$ of individual motor threshold, $500 \mathrm{~ms}$, $20 \mathrm{~Hz}$ ) were delivered - when required by the experimental eventrelated design - simultaneously with picture presentation (Fig. 1b). The same intensity and timing of rTMS was used for sham stimulation. In this case, the coil was still centred on P3 and P4, but it was held perpendicular to the scalp surface, so that scalp contact and discharging noise were quite similar to those for active stimulation, but the induced magnetic field did not activate cortical neurons.

This procedure was used for the PC-rTMS group, and was exactly the same of that used in the retrospectively selected subjects who formed the PFC-rTMS group (Rossi et al., 2001, 2004).

\section{Control experiment 1}

In order to test whether rTMS of the PCs had intensity-dependent effects on memory performance, an additional group of ten healthy subjects (again matched for age, gender, handedness and basal memory performance in the Baseline condition) was tested (see Table 1 for demographics). These subjects (or 'PC-rTMS-120\% group') received rTMS on the same scalp sites, at the same frequency, with the same experimental timing, but with an intensity of stimulation of $120 \%$ of the individual motor threshold.

\section{Control experiment 2}

In order to test whether the applied rTMS train (with the lower intensity, at $90 \%$ of the individual threshold) impaired specific processes of visuospatial attention, an additional 12 subjects (five males) were studied. These were again matched for age (mean 28.5 years, SD 1.6 years), handedness (Oldfield score 60-100\%, mean $93 \%$ ) and scholarity with those included in the other groups. Their sight was normal or corrected-to-normal.

\section{Experimental task}

The subjects kept their forearms resting on the armchairs, with the right index finger resting between two buttons of a mouse, connected to a computer monitor placed in front of them at a distance of about $100 \mathrm{~cm}$. The cue stimulus was a white circle (diameter of about $0.5^{\circ}$ of visual angle) appearing at $6^{\circ}$ right or left of the background central white cross (diameter of $0.5^{\circ}$ ). The cue stimulus was preceded and followed by a masking visual stimulus formed by two ' $\mathrm{Xs}$ ' (about $0.8^{\circ}$ ), located at $6^{\circ}$ right and left of the central white cross. The sequence of the visual stimuli was as follows: (i) the masking stimulus 'Xs' lasting $5.5 \mathrm{~s}$; (ii) the cue stimulus 'small circle' appearing on the right or left $(50 \%)$ monitor side for $500 \mathrm{~ms}$; (iii) the masking stimulus 'Xs' lasting about $2 \mathrm{~s}$; (iv) a go (target) stimulus lasting about $500 \mathrm{~ms}$. The go stimulus was a small green circle with a diameter of about $0.5^{\circ}$, which appeared at $6^{\circ}$ right or left of the central white cross. In $80 \%$ of the cases the go stimulus was given to the same side of the cue stimulus (valid trials) (see Posner et al., 1980).

The subjects had to press the left (or right) mouse button if the go stimulus appeared on the left (or right) monitor side. The computer receiving the mouse inputs registered the corresponding reaction time (RT) and the side of the button pressed.

The rTMS train, delivered during the cue stimulus presentation, was the same as used for the encoding/retrieval protocols $(20 \mathrm{~Hz}, 500 \mathrm{~ms}$,
$90 \%$ of the individual motor threshold) and was applied over the same left or right parietal areas (P3 or P4 of the international EEG 10-20 montage). The same coil orientation was used. As a control, this paradigm was replicated using a left or right sham stimulation, as previously described. Each of the four sessions (active and sham rTMS on P3 and P4) included four blocks of 25 trials. For the statistical analysis we averaged for each subject the RT during all valid trials.

\section{Data analysis}

\section{Main experiments and control experiment 1}

Between-groups demographic characteristics were evaluated by ANOVA (age), chi-squared (gender) and Kruskal-Wallis (handedness). To analyse performance data, ANOVA for repeated measures was applied to hits-false alarms, instead of $\mathrm{d}^{\prime}$ and $\mathrm{C}$, as previously done (Rossi et al., 2001). This because we observed that many subjects of the present study did not make any false alarms or any recognition error in baseline and sham conditions. In this case, $\mathrm{C}$ and $\mathrm{d}^{\prime}$ can be computed only assuming a small proportion of errors, in order to avoid \pm infinite values. This proportion should be arbitrarily small and the choice could affect the results.

A first analysis addressed the differences between the two groups of subjects (i.e. those who received rTMS on the DLPFCs and on the PCs at $90 \%$ of the individual threshold) across the six experimental conditions. This was done by means of a two-way ANOVA (Condition as within-subjects factor, Site of stimulation as between-subjects factor) with Greenhouse-Geisser corrections when necessary, and followed by planned comparisons specifically addressing the differences of L-Enc and R-Ret conditions between the PFC-rTMS and PCrTMS groups.

A second analysis, addressing specifically the HERA model and its relationships with the site of stimulation, was based on a three-way ANOVA for repeated measures with Hemisphere (right vs. left) and rTMS Stimulation (delivered in encoding vs. retrieval) as withinsubjects factors and Site of stimulation (PFC vs. PC) as betweensubjects factor. Finally, ANOVA was applied to compare the cognitive performance between the PC-rTMS and PC-rTMS-120\% groups.

\section{Control experiment 2}

The Reaction Time (dependent variable) was analysed by the ANOVA for repeated measures. Mauchley's test, evaluating the sphericity assumption and correction of the degrees of freedom, was used following the Greenhouse-Geisser procedure. Duncan's test was used for post-hoc comparisons $(P<0.05)$. The ANOVA design included the factors Hemisphere (left, right) and Condition (Sham, Active).

\section{Results}

No side-effects of stimulation were reported. rTMS, even when delivered on parietal sites at $120 \%$ of the individual motor threshold, did not evoke visible muscular responses in any subject.

Because age was very similar in the two groups (Table 1) and no subject was older than 40 years [when functional asymmetry was demonstrated to be age-dependent (Rossi et al., 2004)], its effect was not taken into account.

Table 2 summarizes behavioural results (i.e. hits and hits-false alarms) in the subjects studied. The ANOvA with 'Condition' (six levels) and 'Site of stimulation' (two levels) showed a significant interaction $\left(F_{4.1,74.6}=2.731, P=0.034\right)$, indicating the presence of 
TABLE 2. Percentage of hits and false alarms in the different experimental conditions for the three groups of subjects

\begin{tabular}{|c|c|c|c|c|c|c|}
\hline \multirow[b]{2}{*}{ Experimental blocks } & \multicolumn{3}{|l|}{ Hits $(\%)$} & \multicolumn{3}{|c|}{ Hits (\%) - False alarms (\%) } \\
\hline & $\begin{array}{l}\text { PFC } \\
(n=10)\end{array}$ & $\begin{array}{l}\text { PC- } 90 \% \\
(n=10)\end{array}$ & $\begin{array}{l}\text { PC- } 120 \% \\
(n=10)\end{array}$ & $\begin{array}{l}\mathrm{PFC} \\
(n=10)\end{array}$ & $\begin{array}{l}\text { PC- } 90 \% \\
(n=10)\end{array}$ & $\begin{array}{l}\text { PC- } 120 \% \\
(n=10)\end{array}$ \\
\hline R-Enc & $68 \pm 21$ & $53 \pm 26$ & $54 \pm 20$ & $43 \pm 18$ & $39 \pm 23$ & $19 \pm 24$ \\
\hline L-Enc & $48 \pm 25$ & $51 \pm 25$ & $50 \pm 13$ & $30 \pm 17$ & $41 \pm 25$ & $15 \pm 28$ \\
\hline Sham & $79 \pm 17$ & $71 \pm 26$ & $68 \pm 14$ & $50 \pm 22$ & $49 \pm 21$ & $34 \pm 19$ \\
\hline R-Ret & $49 \pm 24$ & $65 \pm 23$ & $58 \pm 20$ & $13 \pm 16$ & $44 \pm 28$ & $28 \pm 40$ \\
\hline L-Ret & $74 \pm 25$ & $61 \pm 24$ & $59 \pm 18$ & $46 \pm 24$ & $40 \pm 28$ & $24 \pm 15$ \\
\hline Baseline & $66 \pm 26$ & $61 \pm 27$ & $73 \pm 19$ & $48 \pm 28$ & $46 \pm 30$ & $39 \pm 18$ \\
\hline
\end{tabular}

Data are given as means \pm SD.Group PFC, subjects who received rTMS on the dorsolateral prefrontal cortex; groups PC, subjects who received rTMS on the parietal cortex at $90 \%$ or at $120 \%$ of individual motor thresholds. The six encoding/retrieval blocks were: R-Enc, right rTMS in encoding, no stimulation in retrieval; L-Enc, left rTMS in encoding, no stimulation in retrieval; Sham, sham rTMS (left in encoding and right in retrieval); R-Ret, right rTMS in retrieval, no stimulation in encoding; L-Ret, left rTMS in retrieval, no stimulation in encoding; Baseline, absence of stimulation.

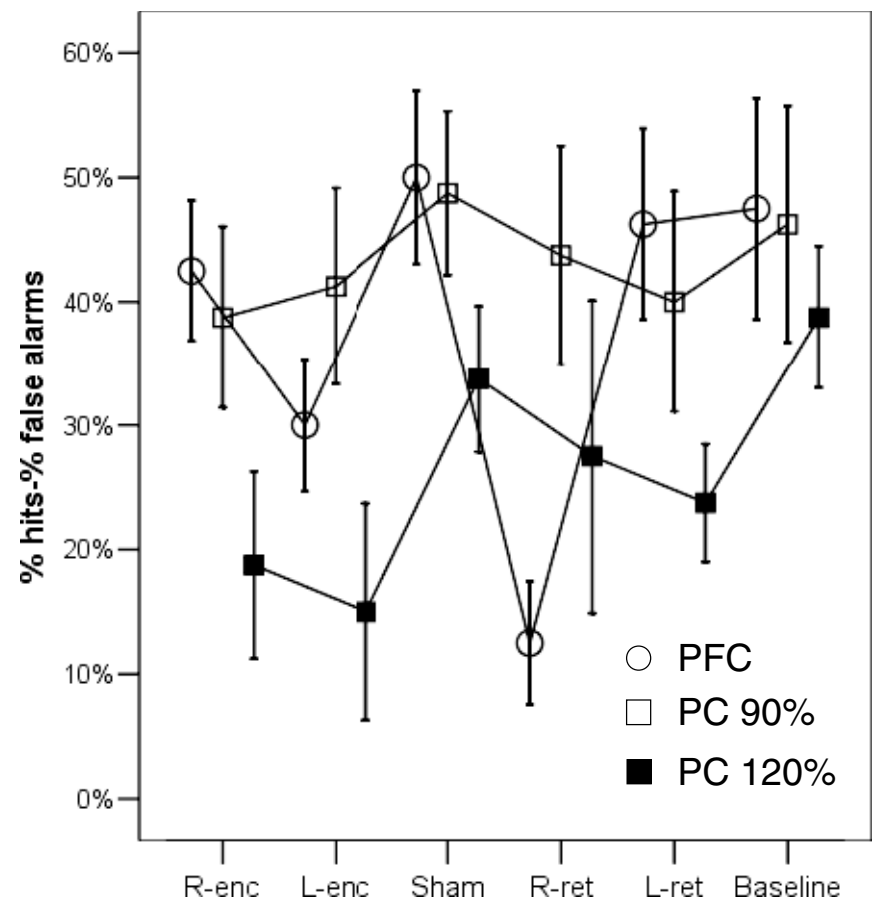

FIG. 2. Summary of the behavioural results (percentage of hits - percentage of false alarms; \pm 1 SEM) in the two groups of subjects who were the core of the study (i.e. PFC-rTMS and PC-rTMS at $90 \%$ of motor threshold) across the six experimental conditions. The additional values (black squares) refer to subjects who received PC-rTMS at higher intensity. Note the similar performance in baseline (for the three groups) and sham blocks (for PFCrTMS and PC 90\%-rTMS). Prefrontal cortex rTMS worsened behavioural performance more than PC-rTMS (at both intensities) in R-Ret and, to a lesser extent, in L-Enc vs. PC 90\%-rTMS.

a lack of parallelism of the two profiles across conditions (Fig. 2). This was due to the strongly significant 'Condition' effect in the PFC-rTMS group $\left(\mathrm{F}_{3.1,27.7}=5.454, P=0.004\right)$ and to the null effect in the PC-rTMS group $\left(F_{3.0,27.4}=0.413, P=0.748\right)$. The matching procedure based on behavioural performance in baseline condition (resulting in a $P$-value of 0.925 ) also produced an overlap in terms of hits-false alarm rates in the sham condition $(P=0.898)$. The other planned comparisons between the two groups indicated that the only significant difference was in the R-Ret condition $(P=0.006)$.

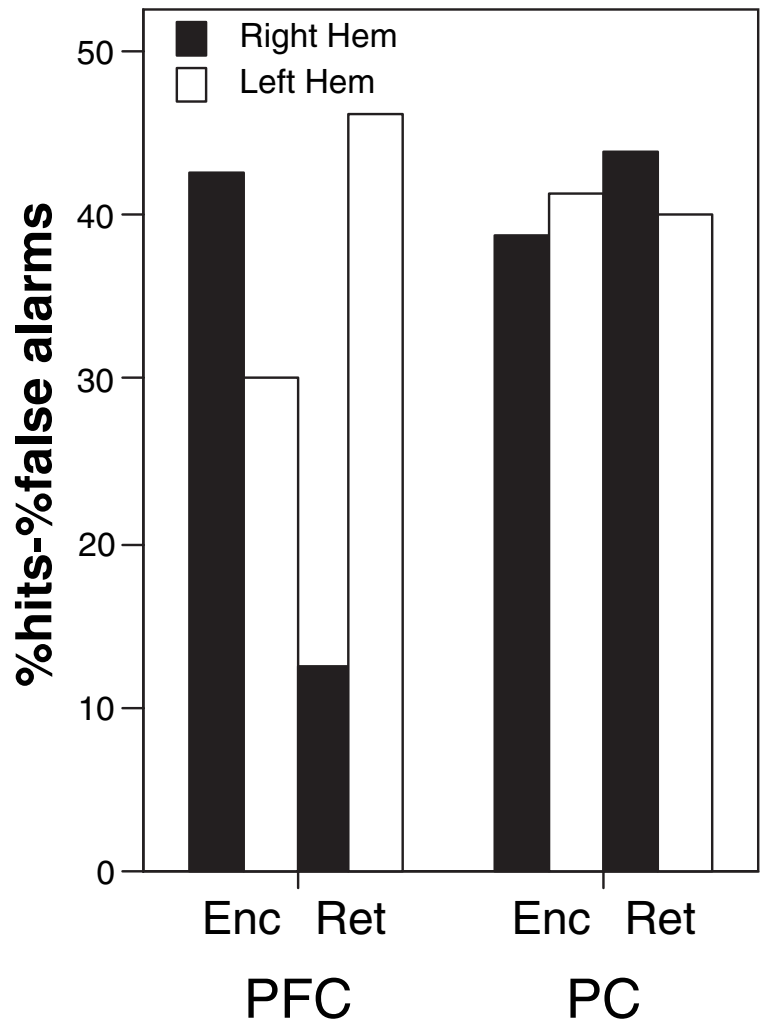

FIG. 3. The HERA (Hemispheric Encoding Retrieval Asymmetry) pattern, which is clearly evident in the PFC-rTMS group, is absent in the PC-rTMS group.

Focusing the ANOVA on the HERA model, a significant triple interaction occurred $\left(F_{1,18}=8.418, P=0.010\right)$. This finding is the result of the HERA pattern in the sample drawn from the population that received rTMS on the PFC $\left(F_{1,9}=23.65, P=0.001\right)$, and of the lack of such asymmetry in the PC-rTMS group $\left(F_{1,9}=0.165\right.$, $P=0.694$ ) (Fig. 3).

In control experiment 1 , a close descriptive inspection of recognition performance in R-Ret showed more variable effects (i.e. higher standard deviations of hits-false alarms) than that observed in the PC-rTMS group (Table 2, Fig. 2). However, ANOvA indicated that the performance was significantly lower in the PC-rTMS-120\% group 


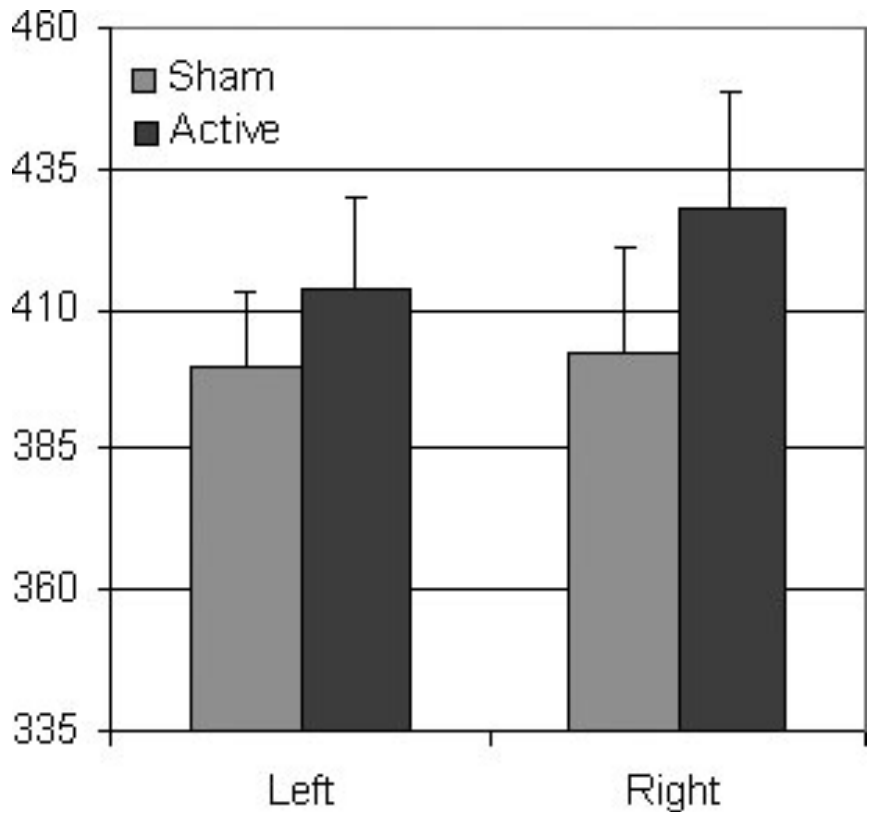

FIG. 4. Graphical representation of the RTs in control experiment 2 (12 subjects pooled), based on the Posner's test of visuospatial attention. Active rTMS delivered on the left (mean $414 \mathrm{~ms}$, SEM $15.7 \mathrm{~ms}$ ) or right (mean $428 \mathrm{~ms}$, SEM $20.1 \mathrm{~ms}$ ) IPS increases reaction times more than the corresponding sham stimulation (left: mean $399 \mathrm{~ms}$, SEM $13.9 \mathrm{~ms}$; right: $402 \mathrm{~ms}$, SEM $18.8 \mathrm{~ms}$ ). Rection times are significantly more delayed by active right rTMS than active left stimulation. Statistics are given in the text.

$\left(F_{1,18}=5.960, P=0.025\right)$, with the exception of the baseline block in which no stimulation occurred, but with profiles across the other conditions substantially parallel in the two groups $\left(F_{5,90}=0.379\right.$, $P=0.862)$. Again, any significant differences between conditions were apparent. This overall non-specific memory impairment, including the sham condition, suggests that the specific left and right rTMS effects on encoding and retrieval, respectively, are not intensitydependent.

In control experiment 2, ANOVA analysis of the RTs showed a statistically significant interaction $\left(F_{1,11}=5,27, P<0.0424\right)$ among all factors such as Hemisphere (left, right), and Condition (active, sham). Post-hoc comparisons (Fig. 4) showed that both left and right active rTMS slowed RTs with respect to sham stimulation $(P<0.002)$. Furthermore, the RTs evaluated during the right active stimulations were longer than during the left stimulation $(P<0.006)$.

\section{Discussion}

The current investigation was aimed to address directly, using rTMS, the functional role of a subregion of the PCs (i.e. the intraparietal sulcus) during episodic encoding and retrieval of visual scenes. The lack of significant specific recognition errors induced by the eventrelated rTMS interference suggests that the functional relevance of these left and right PC subregions, differently from that of DLPFCs, is poorly correlated with a successful completion of the episodic memory task, given that neither encoding nor retrieval mechanisms were significantly disrupted by left or right PC-rTMS. These negative results appear to be robust, as specific behavioural changes both in encoding and in retrieval were lacking even when the interference with rTMS was carried out with a higher intensity of stimulation. Indeed, increasing the intensity of rTMS (i.e. control experiment 1) produced an overall non-specific memory impairment (see Table 2 and Fig. 2, hits-false alarms), which was similar in all experimental conditions, including the sham. This suggests a non-specific interference effect of rTMS on the demanding cognitive task, probably linked to a merely distraction mechanism resulting from high discharge noise.

Conversely, the same low-intensity rTMS train (i.e. $90 \%$ of the motor threshold) of the PC group, delivered on the same scalp regions, was clearly able specifically and asymmetrically to interfere with RTs in the context of a purely visuospatial attentive task (Posner et al., 1980).

Before discussing the possible implications of the present results and their possible relevance for our current knowledge of the physiological mechanisms underlying episodic memory, the following methodological key points should be taken into account:

1 A similar event-related rTMS interference approach, using the same set of memoranda and carried out on a fully matched sample of subjects, was successfully utilized to demonstrate directly the functional relevance of DLPFCs in episodic memory performance, both in young adults and in normal ageing (Rossi et al., 2001, 2004).

2 As a general rule for event-related rTMS studies, scalp stimulation sites for the intraparietal sulcus were chosen on the basis of independent neuroimaging studies (Walsh \& Pascual-Leone, 2003), which provide the best available spatial resolution to detect eloquent regions for a given task. Indeed, brain activity in these PC regions had been previously found to be engaged consistently in episodic encoding and retrieval (Mottaghy et al., 1999; Cabeza et al., 2003; Fujii et al., 2004; Hayes et al., 2004; Mayes et al., 2004; Wagner et al., 2005).

3 The accuracy of TMS coil positioning (SofTax system) can be estimated to be of the order of less than $1 \mathrm{~cm}$ (Herwig et al., 2001). Nevertheless, the high intensity of stimulation used in the PC-rTMS-120\% group strongly minimizes the possibility of an insufficient interference effect due to a mismatch between the scalp site and the underlying anatomy. We should be aware, however, that both the spatial selectivity and the intimate mechanisms by which rTMS works are still not wholly understood (see Walsh \& PascualLeone, 2003; Rossi \& Rossini, 2004).

4 The same scalp positions have been previously used in TMS studies, using intensities of stimulation similar to the lower one adopted in the present study (i.e. $90 \%$ of the individual motor threshold), to interfere with cognitive functions associated with activity of the intraparietal sulcus region, such as sensory perception (Oliveri et al., 1999, 2000), mental rotation (Bestmann et al., 2002), grasping (Tunik et al., 2005) or visuospatial and verbal working memory (Oliveri et al., 2001; Mottaghy et al., 2002; Koch et al., 2005). Moreover, results from control experiment 2 are in line with previous rTMS studies, which suggested that the right parietal lobe plays a significant role in purely visuospatial attentional processes (Walsh et al., 1999; Hilgetag et al., 2001).

5 According to previous studies on DLPFC function based on the same experimental timing and setting (Rossi et al., 2001, 2004), the temporal extent to which the applied rTMS train is likely to be effective in disrupting encoding and retrieval processing is at least $1.5 \mathrm{~s}$, i.e. the time including that of picture display and the following yes/no motor response. Such duration is therefore largely sufficient to include all the temporal dynamics of parietal activations, as determined from event-related potentials (ERP) studies, during similar memory tasks (i.e. old/new successful recollection), which is known to emerge approximately $400 \mathrm{~ms}$ post-stimulus onset and to last about $400-600 \mathrm{~ms}$ (see Allan et al., 1998; Paller \& Wagner, 2002). 
Keeping in mind all the abovementioned points, it is very unlikely that the lack of significant detrimental effects induced by rTMS of the PCs on memory performance could be due to experimental or methodological biases. Note that the same rTMS train used for encoding/retrieval protocols was able to interfere with a purely visuospatial attentional task in the control experiment 2 .

The question of whether the PCs play a relevant role in episodic memory operations is still open. The present rTMS findings seem to challenge the results of recent neuroimaging and electrophysiological studies, which detected metabolic/cerebral blood flow increases (Henson et al., 1999; Mottaghy et al., 1999; Konishi et al., 2000; Fujii et al., 2004; Hayes et al., 2004; Mayes et al., 2004) and the presence of gamma EEG oscillations (Babiloni et al., 2004) in specific PC regions during successful episodic retrieval. The differential sensitivity of neuroimaging methods to the diverse components of long-term episodic memory processes, as well as differences in experimental material and settings, can plausibly at least in part explain such discrepancies.

The present findings cannot offer definite proof as to why the stimulated PC subregions do not participate to a useful degree in encoding and retrieval processes in this experimental setting. However, it should be considered that the correlational nature of neuroimaging and electrophysiological findings leaves open the possibility that such activations could also be due to additional brain processes that are simply associated with, but are not crucial for, the memory challenge. Of note, it has been recently suggested (Cabeza et al., 2003) that several brain regions including $\mathrm{PFC}$, parietal, anterior cingulate and thalamic regions, known to be critical components of a distributed brain network for attention (Mesulam, 1990; Posner \& Petersen, 1990), become active during episodic retrieval. Such widespread attentional processes, which are compatible with the post-retrieval monitoring hypothesis (Cabeza et al., 2003), can be reasonably translated to the encoding phase, which also requires attention to - and successive elaboration of - the presented memoranda. Therefore, the parietal activations accompanying episodic memorization processes could reflect the engagement of attentional networks during encoding and retrieval. Interference with a single 'node' of the network, although sufficient to interfere with a spatial attention task (i.e. control experiment 2), does not result in the overt disruption of performance in the visual memory task, in which an overall analysis of a visual scene is required, rather than selective attention to the details. Anticipating the timing of rTMS with respect to memoranda presentation might address this point more directly, by considering that studies on memory-guided saccades (i.e. a reflection of spatial memory) suggest that the posterior PC is involved at a very initial stage (i.e. less than $300 \mathrm{~ms}$ ) of visuospatial integration (Pierrot-Deseilligny et al., 2002).

The current results, however, do not exclude that other regions located lateral and posterior to the intraparietal sulcus (see Wheeler \& Buckner, 2004), such as the precuneus, could actively participate in episodic retrieval, as suggested by previous neuroimaging studies (Buckner et al., 1996; Krause et al., 1999; Mottaghy et al., 1999; Cabeza \& Nyberg, 2000; Kohler et al., 2002; Cabeza et al., 2003; Mayes et al., 2004; Wheeler \& Buckner, 2004). It is unlikely that the interference of focal rTMS delivered on the scalp overlying the intraparietal sulcus would spread to such relatively distant sites. This is strongly supported by the fact that previous studies demonstrated distinct neuophysiological and behavioural effects of rTMS applied to even closer cortical regions, such as primary motor and premotor areas (Munchau et al., 2002; Cincotta et al., 2004) or primary and nonprimary visual cortical areas (i.e. V1 and V5, Silvanto et al., 2005).

Another, not mutually exclusive, hypothesis that can be taken into account to explain the absence of significant rTMS interference of the stimulated region(s) (i.e. the left and right intraparietal sulcus) on encoding and retrieval mechanisms is that these areas could actually directly participate in episodic learning and memory, but at a lower level and in a complex, hierarchically organized, multinode neural network, in which medial temporal lobe structures (Cabeza et al., 2003) and PFCs (Babiloni et al., 2006) play a leading role. Should this be the case, then it is not surprising that interference rTMS effects of PCs are not detrimental to memory performance, as the function of the main nodes of the whole network remain free from interference. However, the lack of evidence for overt detrimental effects of parietal lesions on episodic memory militates against this interpretation.

In conclusion, the present findings suggest that activity of intraparietal sulcus, unlike that of DLPFC, does not subserve at a useful degree memory encoding and retrieval, at least under these specific experimental conditions, while it plays a causal role in purely visuospatial attentive processes. It can be hypothesized that PC activity accompanying episodic memory (as revealed by positron emission tomography, functional magnetic resonance and EEG) could be associated with parallel and more complex attentional and visuospatial integrative processes and that regional rTMS interference could be compensated for by other nodes of the distributed attentional system.

Moreover, complex cognitive phenomena - as in the case of episodic memory - provide a fitting example as to how underlying physiological mechanisms cannot be fully disclosed by investigations based on a single technique. Future studies on episodic learning and memory will take advantage of a true multimodal approach to the working brain, with the aim of combining the relative advantages provided by cerebral blood flow-based neuroimaging techniques, sush as positron emission tomography or functional magnetic resonance (i.e. spatial details), by electrophysiological methods, such as EEG and ERPs (i.e. temporal resolution) and by the behavioural interference of rTMS (to prove causal relationships).

\section{Acknowledgements}

We thank F. Carducci for the development and update of the SofTaxic software and A. Brancucci, M. Ulivelli, S. Bartalini and A. De Capua for experimental help. The work was partly supported by Ministero della Sanità (Progetto Finalizzato 1999).

\section{Abbreviations}

DLPFC, dorsolateral prefrontal cortex; EEG, electroencephalography; ERP, event-related potentials; HERA, Hemispheric Encoding Retrieval Asymmetry; PC, parietal cortex; PFC, prefrontal cortex; RT, reaction time; rTMS, repetitive transcranial magnetic stimulation.

\section{References}

Alexander, M.P., Stuss, D.T. \& Fansabedian, N. (2003) California Verbal Learning Test: performance by patients with focal frontal and non-frontal lesions. Brain, 126, 1493-1150.

Allan, K., Wilding, E.L. \& Rugg, M.D. (1998) Electrophysiological evidence for dissociable processes contributing to recollection. Acta Psychol. (Amst.), 98, 231-252.

Babiloni, C., Babiloni, F., Carducci, F., Cappa, S.F., Cincotti, F., Del Percio, C., Miniussi, C., Moretti, D.V., Pasqualetti, P., Rossi, S., Sosta, K. \& Rossini, P. (2004) Human cortical EEG rhythms during long-term episodic memory. A high-resolution EEG study of the HERA model. Neuroimage, 21, 1576-1584.

Babiloni, C., Vecchio, F., Cola, B., Babiloni, F., Rossi, S., Miniussi, C. \& Rossini, P.M. (2006) Functional frontoparietal connectivity during encoding and retrieval processes follows the HERA model. A high-resolution EEG study. Brain Res. Bull., 68, 203-212.

Baddeley, A., Conway, M. \& Aggleton, J. (eds) (2001) Episodic memory. Phil. Trans. R. Soc. London B, 356, 1341-1515. 
Bestmann, S., Thilo, K.V., Sauner, D., Siebner, H.R. \& Rothwell, J.C. (2002) Parietal magnetic stimulation delays visuomotor mental rotation at increased processing demands. Neuroimage, 17, 1512-1520.

Buckner, R.L., Raichle, M.E., Miezin, F.M. \& Petersen, S.E. (1996) Functional anatomic studies of memory retrieval for auditory words and visual pictures. J. Neurosci., 16, 6219-6235.

Buckner, R.L. \& Wheeler, M.E. (2001) The cognitive neuroscience of remembering. Nat. Neurosci. Rev., 2, 624-634.

Cabeza, R., Dolcos, F., Prince, S.E., Rice, H.J., Weissman, D.H. \& Nyberg, L. (2003) Attention-related activity during episodic memory retrieval: a crossfunction fMRI study. Neuropsychologia, 41, 390-399.

Cabeza, R. \& Nyberg, L. (2000) Imaging Cognition II: an empirical review of 275 PET and fMRI studies. J. Cogn. Neurosci., 12, 1-47.

Cincotta, M., Borgheresi, A., Balestrieri, F., Giovannelli, F., Rossi, S., Ragazzoni, A., Zaccara, G. \& Ziemann, U. (2004) Involvement of the human dorsal premotor cortex in unimanual motor control: an interference approach using transcranial magnetic stimulation. Neurosci. Lett., 367, 189-193.

De Renzi, E. \& Nichelli, P. (1975) Verbal and non-verbal short-term memory impairment following hemispheric damage. Cortex, 11, 341-354.

Fletcher, P.C. \& Henson, R.N.A. (2001) Frontal lobes and human memory. Insights from functional neuroimaging. Brain, 124L, 849-881.

Floel, A., Poeppel, D., Buffalo, E.A., Braun, A., Wu, C.W., Seo, H.J., Stefan, K., Knecht, S. \& Cohen, L.G. (2004) Prefrontal cortex asymmetry for memory encoding of words and abstract shapes. Cereb. Cortex, 14, 404-409.

Fujii, T., Suzuki, M., Okuda, J., Othake, H., Tanji, K., Yamagouchi, K., Itoh, M. \& Yamadori, A. (2004) Neural correlates of context-memory with realworld events. Neuroimage, 21, 1596-1603.

Habib, R., Nyberg, L. \& Tulving, E. (2003) Hemispheric asymmetries of memory: the HERA model revisited. Trends Cogn. Sci., 7, 241-245.

Hayes, S.M., Ryan, L., Schnyer, D.M. \& Nadel, L. (2004) An fMRI study of episodic memory: retrieval of object, spatial, and temporal information. Behav. Neurosci., 118, 885-896.

Henson, R.N., Rugg, M.D., Shallice, T., Josephs, O. \& Dolan, R.J. (1999) Recollection and familiarity in recognition memory: an event-related functional magnetic resonance imaging study. J. Neurosci., 19, 3962-3972.

Herwig, U., Schonfeldt-Lecuona, C., Wunderlich, A.P., von Tiesenhausen, C., Thielscher, A., Walter, H. \& Spitzer, M. (2001) The navigation of transcranial magnetic stimulation. Psychiatry Res., 108, 123-131.

Hilgetag, C.C., Theoret, H. \& Pascual-Leone, A. (2001) Enhanced visual spatial attention ipsilateral to rTMS-induced 'virtual lesions' of human parietal cortex. Nat. Neurosci., 4, 953-957.

Kesner, R.P. \& Rogers, J. (2004) An analysis of independence and interactions of brain substrates that subserve multiple attributes, memory systems, and underlying processes. Neurobiol. Learn. Mem., 82, 199-215.

Koch, G., Oliveri, G., Torriero, S. \& Caltagirone, C. (2005) Modulation of excitatory and inhibitory circuits for visual awareness in the human right parietal cortex. Exp. Brain Res., 160, 510-516.

Kohler, S., Crane, J. \& Milner, B. (2002) Differential contributions of the parahippocampal place area and the anterior hippocampus to human memory for scenes. Hippocampus, 12, 718-723.

Kohler, S., Paus, T., Buckner, R.L. \& Milner, B. (2004) Effects of left inferior prefrontal stimulation on episodic memory formation: a two-stage fMRIrTMS study. J. Cogn. Neurosci., 16, 178-188.

Konishi, S., Wheeler, M.E., Donaldson, D.I. \& Buckner, R.L. (2000) Neural correlates of episodic retrieval success. Neuroimage, 12, 276-286.

Krause, B.J., Schmidt, D., Mottaghy, F.M., Taylor, J., Halsband, U. \& Herzog, H. (1999) Episodic retrieval activates the precuneus irrespective of the imagery content of word pairs associates: a PET study. Brain, 122, 225-263.

Mayes, A.R., Montaldi, D., Spencer, T.J. \& Roberts, N. (2004) Recalling spatial information as a component of recently and remotely acquired episodic or semantic memory: an fMRI study. Neuropsychology, 18, 426-441.

Mesulam, M.M. (1990) Large-scale neurocognitive networks and distributed processes of attention, language, and memory. Ann. Neurol., 28, 597-613.

Miniussi, C., Cappa, S.F., Sandrini, M., Rossini, P.M. \& Rossi, S. (2003) The causal role of the prefrontal cortex in episodic memory as demonstrated with rTMS. Clin. Neurophysiol., 56 (Suppl.), 312-320.

Mottaghy, F.M., Doring, T., Muller-Gartner, H.W., Topper, R. \& Krause, B.J. (2002) Bilateral parieto-frontal network for verbal working memory: an interference approach using repetitive transcranial magnetic stimulation (rTMS). Eur. J. Neurosci., 16, 1627-1632.

Mottaghy, F.M., Shah, N.J., Krause, B.J., Schmidt, D., Halsband, U., Jäncke, L. \& Müller-Gärtner, H.W. (1999) Neuronal correlates of encoding and retrieval in episodic memory during a paired-word association learning task: a functional magnetic resonance imaging study. Exp. Brain Res., 128, 332 342 .

Munchau, A., Bloem, B.R., Irlbacher, K., Trimble, M.R. \& Rothwell, J.C. (2002) Functional connectivity of human premotor and motor cortex explored with repetitive transcranial magnetic stimulation. J. Neurosci., 22, 554-561.

Oliveri, M., Rossini, P.M., Filippi, M.M., Traversa, R., Cicinelli, P., Palmieri, M.G., Pasqualetti, P. \& Caltagirone, C. (2000) Time-dependent activation of parieto-frontal networks for directing attention to tactile space. A study with paired transcranial magnetic stimulation pulses in right-brain-damaged patients with extinction. Brain, 123, 1939-1947.

Oliveri, M., Rossini, P.M., Pasqualetti, P., Traversa, R., Cicinelli, P., Palmieri, M.G., Tomaiuolo, F. \& Caltagirone, C. (1999) Interhemispheric asymmetries in the perception of unimanual and bimanual cutaneous stimuli. A study using transcranial magnetic stimulation. Brain, 122, 1721-1729.

Oliveri, M., Turriziani, P., Carlesimo, G.A., Koch, G., Tomaiuolo, F., Panella, M. \& Caltagirone, C. (2001) Parieto-frontal interactions in visual-object and visual-spatial working memory: evidence from transcranial magnetic stimulation. Cereb. Cortex, 11, 606-618.

Paller, K.A. \& Wagner, A.D. (2002) Observing the transformation of experience into memory. Trends Cogn. Sci., 6, 93-102.

Pierrot-Deseilligny, C., Muri, R.M., Rivaud-Pechoux, S., Gaymard, B. \& Ploner, C.J. (2002) Cortical control of spatial memory in humans: the visuooculomotor model. Ann. Neurol., 52, 10-19.

Posner, M.I. \& Petersen, S.F. (1990) The attention system of the human brain. Annu. Rev. Neurosci., 13, 25-42.

Posner, M.I., Snyder, C.R. \& Davidson, B.J. (1980) Attention and the detection of signals. J. Exp. Psychol, 109, 160-174.

Rami, L., Gironell, A., Kulisevsky, J., Garcia-Sanchez, C., Berthier, M. \& Estevez-Gonzalez, A. (2003) Effects of repetitive transcranial magnetic stimulation on memory subtypes: a controlled study. Neuropsychologia, 41, 1877-1883.

Rossi, S., Cappa, S.F., Babiloni, C., Pasqualetti, P., Miniussi, C., Carducci, F., Babiloni, F. \& Rossini, P.M. (2001) Prefrontal cortex in long-term memory: an 'interference' approach using magnetic stimulation. Nat. Neurosci., 4, 948-952.

Rossi, S., Miniussi, C., Pasqualetti, P., Babiloni, C., Rossini, P.M. \& Cappa, S.F. (2004) Age-related functional changes of prefrontal cortex in long-term memory. A repetitive transcranial magnetic stimulation (rTMS) study. J. Neurosci., 24, 7939-7944.

Rossi, S. \& Rossini, P.M. (2004) TMS in cognitive plasticity and the potential for rehabilitation. Trends Cogn. Sci., 8, 273-279.

Rugg, M., Otten, L.J. \& Henson, R.N. (2002) The neural basis of episodic memory: evidence from functional neuroimaging. Phil. Trans. R. Soc. London B, 357, 1097-1110.

Sandrini, M., Cappa, S.F., Rossi, S., Rossini, P.M. \& Miniussi, C. (2003) The role of prefrontal cortex in verbal episodic memory: rTMS evidence. J. Cogn. Neurosci., 15, 855-861.

Silvanto, J., Cowey, A., Lavie, N. \& Walsh, V. (2005) Striate cortex (V1) activity gates awareness of motion. Nat. Neurosci., 8, 143-144.

Simons, J.S. \& Spiers, H.J. (2003) Prefrontal and medial temporal lobe interactions in long-term memory. Nat. Rev. Neurosci., 4, 637-648.

Squire, L.R., Clark, R.E. \& Knowlton, B.J. (2001) Retrograde amnesia. Hippocampus, 11, 50-55.

Tulving, E. (2002) Episodic memory: from mind to brain. Annu. Rev. Psychol., 53, $1-25$.

Tunik, E., Frey, S.H. \& Grafton, S.T. (2005) Virtual lesions of the anterior intraparietal area disrupt goal-dependent on-line adjustments of grasp. Nat. Neurosci., 8, 505-511.

Valenstein, E., Bowers, D., Verfellie, M., Heilman, K.M., Day, A. \& Watson, R.T. (1987) Retrosplenial amnesia. Brain, 110, 1631-1646.

Wagner, A.D., Shannon, B.J., Kahn, I. \& Buckner, R. (2005) Parietal lobe contributions to episodic memory retrieval. Trends Cogn. Sci., 9, 445-453.

Walsh, V. \& Cowey, A. (2000) Transcranial magnetic stimulation and cognitive neuroscience. Nat. Rev. Neurosci., 1, 73-79.

Walsh, V., Ellison, A., Ashbridge, E. \& Cowey, A. (1999) The role of parietal cortex in visual attention-hemispheric asymmetries and the effects of learning: a magnetic stimulation study. Neuropsychologia, 37, 245-251.

Walsh, V. \& Pascual-Leone, A. (2003) Neurochronometrics of Mind: Transcranial Magnetic Stimulation in Cognitive Science. MIT Press, Cambridge, MA.

Wheeler, M.E. \& Buckner, R.L. (2004) Functional-anatomic correlates of remembering and knowing. Neuroimage, 21, 1337-1349. 